\title{
Quantum Memory for Light
}

\author{
A. E. Kozhekin, K. Mølmer and E. Polzik \\ Institute of Physics and Astronomy, University of Aarhus, Ny Munkegade, DK-8000 Aarhus C, Denmark
}

(March 8, 2018)

\begin{abstract}
We propose an efficient method for mapping and storage of a quantum state of propagating light in atoms. The quantum state of the light pulse is stored in two sublevels of the ground state of a macroscopic atomic ensemble by activating a synchronized Raman coupling between the light and atoms. We discuss applications of the proposal in quantum information processing and in atomic clocks operating beyond quantum limits of accuracy. The possibility of transferring the atomic state back on light via teleportation is also discussed.
\end{abstract}

42.50.Lc, 03.67.-a, 42.50.Dv, 42.50.Ct, 06.30.Ft

Light is an ideal carrier of quantum information, but photons are difficult to store for a long time. In order to implement a storage device for quantum information transmitted as a light signal, it is necessary to faithfully map the quantum state of the light pulse onto a medium with low dissipation, allowing for storage of this quantum state. Depending on the particular application of the memory, the next step may be either a (delayed) measurement projecting the state onto a certain basis, or further processing of the stored quantum state, e.g., after a read-out via the teleportation process. The delayed projection measurement is relevant for the security of various quantum cryptography and bit commitment schemes [1]. The teleportation read-out is relevant for full scale quantum computing.

In this Letter we propose a method that enables quantum state transfer between propagating light and atoms with an efficiency up to $100 \%$ for certain classes of quantum states. The long term storage of these quantum states is achieved by utilizing atomic ground states. In the end of the paper we propose an atom-back-to-light teleportation scheme as a read-out method for our quantum memory.

We consider the stimulated Raman absorption of propagating quantum light by a cloud of $\Lambda$ atoms. As shown in the inset of Fig.11, the weak quantum field and the strong classical field are both detuned from the upper intermediate atomic state(s) by $\Delta$ which is much greater than the strong field Rabi frequency $\Omega_{s}$, the width of an upper level $\gamma_{i}$ and the spectral width of the quantum light $\Gamma_{q}$. The Raman interaction "maps" the non-classical features of the quantum field onto the coherence of the lower atomic doublet, distributed over the atomic cloud.

In our analysis we eliminate the excited intermediate states, and we treat the atoms by an effective two-level approximation. We start with the quantum Maxwell-Bloch equations in the lowest order for the slowly varying operator $\hat{Q}: \hat{Q}=\hat{\sigma_{3}} e^{-i\left(\omega_{q}-\omega_{s}\right) t+i\left(k_{q}-k_{s}\right) z}$ (it will be assumed, that $\left(k_{q}-k_{s}\right) L \ll 1$, where $L$ is the length of the atomic cloud, $z$ is the propagation direction, and $\omega_{q, s}$ and $k_{q, s}$ are frequencies and wavevectors of "quantum" and "strong" fields respectively) [2,3]

$$
\begin{aligned}
& \frac{d}{d t} \hat{Q}(z, t)=-i \kappa_{1}^{*} \hat{E}_{q}(z, t) E_{s}^{*}(z, t)-\Gamma \hat{Q}(z, t)+\hat{F}(z, t) \\
& \left(\frac{\partial}{\partial z}+\frac{1}{c} \frac{\partial}{\partial t}\right) \hat{E}_{q}(z, t)=-i \kappa_{2} \hat{Q}(z, t) E_{s}(z, t)
\end{aligned}
$$

$\Gamma$ is the dephasing rate of the $1 \leftrightarrow 3$ coherence which also includes the strong field power broadening $\Gamma_{s} \simeq$ $\omega^{3} \hbar \kappa_{1}^{2}\left|E_{s}\right|^{2} /\left(3 c^{3}\right)$ due to spontaneous Raman scattering [2], $\hat{F}(z, t)$ is the associated quantum Langevin force with correlation function $\left\langle\hat{F}^{*}(z, t) \hat{F}\left(z^{\prime}, t^{\prime}\right)\right\rangle=2 \Gamma / n \delta\left(z-z^{\prime}\right) \delta\left(t-t^{\prime}\right)$, and $\kappa_{1}=\sum_{i} \mu_{1 i} \mu_{3 i} /\left(\hbar^{2} \Delta_{i}\right), \kappa_{2}=2 \pi n \hbar \omega \kappa_{1} / c$, where $\mu_{j i}$ are dipole moments of the atomic transitions and $n$ is the density of the atoms. A one-dimensional wave equation is sufficient to describe the spatial propagation of light in a pencil-shaped sample with a Fresnel number $\mathcal{F}=A / \lambda L$ near unity ( $A$ is the cross-sectional area of the sample and $\lambda$ is the optical wavelength) [3].

If the strong field is not depleted in the process of quantum field absorption and if most of the atomic population stays in the initial level 1, Eqs.(1a 1 (b) can be integrated to get

$$
\begin{aligned}
\hat{Q}(z, \tau) & =e^{-\Gamma \tau} \hat{Q}(z, 0)-e^{-\Gamma \tau} \int_{0}^{z} d z^{\prime} \hat{Q}\left(z^{\prime}, 0\right) \sqrt{\frac{a(\tau)}{z-z^{\prime}}} J_{1}\left(2 \sqrt{a(\tau)\left(z-z^{\prime}\right)}\right) \\
& -i \kappa_{1} \int_{0}^{\tau} d \tau^{\prime} e^{-\Gamma\left(\tau-\tau^{\prime}\right)} \hat{E}_{q}\left(0, \tau^{\prime}\right) E_{s}\left(\tau^{\prime}\right) J_{0}\left(2 \sqrt{z\left(a(\tau)-a\left(\tau^{\prime}\right)\right)}\right)+\int_{0}^{\tau} d \tau^{\prime} e^{-\Gamma\left(\tau-\tau^{\prime}\right)} \hat{F}\left(z, \tau^{\prime}\right) \\
& -\int_{0}^{\tau} d \tau^{\prime} \int_{0}^{z} d z^{\prime} e^{-\Gamma\left(\tau-\tau^{\prime}\right)} \hat{F}\left(z^{\prime}, \tau^{\prime}\right) \sqrt{\frac{a(\tau)-a\left(\tau^{\prime}\right)}{z-z^{\prime}}} J_{1}\left(2 \sqrt{\left(a(\tau)-a\left(\tau^{\prime}\right)\right)\left(z-z^{\prime}\right)}\right)
\end{aligned}
$$




$$
\begin{aligned}
\hat{E}_{q}(z, \tau) & =\hat{E}_{q}(0, \tau)-i \kappa_{2} E_{s}(\tau) e^{-\Gamma \tau} \int_{0}^{z} d z^{\prime} \hat{Q}\left(z^{\prime}, 0\right) J_{0}\left(2 \sqrt{a(\tau)\left(z-z^{\prime}\right)}\right) \\
& -\kappa_{1}^{*} \kappa_{2} E_{s}(\tau) \int_{0}^{\tau} d \tau^{\prime} e^{-\Gamma\left(\tau-\tau^{\prime}\right)} \hat{E}_{q}\left(0, \tau^{\prime}\right) E_{s}^{*}\left(\tau^{\prime}\right) \sqrt{\frac{z}{a(\tau)-a\left(\tau^{\prime}\right)}} J_{1}\left(2 \sqrt{z\left(a(\tau)-a\left(\tau^{\prime}\right)\right)}\right) \\
& -i \kappa_{2} E_{s}(\tau) \int_{0}^{\tau} d \tau^{\prime} \int_{0}^{z} d z^{\prime} e^{-\Gamma\left(\tau-\tau^{\prime}\right)} \hat{F}\left(z^{\prime}, \tau^{\prime}\right) J_{0}\left(2 \sqrt{\left.\left(a(\tau)-a\left(\tau^{\prime}\right)\right)\left(z-z^{\prime}\right)\right)}\right.
\end{aligned}
$$

where $\tau=t-z / c$, and $a(\tau)=\kappa_{1}^{*} \kappa_{2} \int_{0}^{\tau} d \tau^{\prime \prime}\left|E_{s}\left(\tau^{\prime \prime}\right)\right|^{2}$ and $\hat{Q}(z, 0)$ is the initial atomic coherence.

Integrating Eq.(2a) over space we obtain the collective atomic spin operator, which is the atomic variable on which the quantum light field is mapped.

$$
\begin{aligned}
\hat{\mathcal{Q}}_{L}(\tau) \equiv n \int_{0}^{L} d z \hat{Q}(z, \tau) & =n e^{-\Gamma \tau} \int_{0}^{L} d z^{\prime} J_{0}\left(2 \sqrt{a(\tau)\left(L-z^{\prime}\right)}\right) \hat{Q}\left(z^{\prime}, 0\right) \\
& +n \int_{0}^{\tau} d \tau^{\prime} e^{-\Gamma\left(\tau-\tau^{\prime}\right)} \int_{0}^{L} d z^{\prime} J_{0}\left(2 \sqrt{\left(a(\tau)-a\left(\tau^{\prime}\right)\right)\left(L-z^{\prime}\right)}\right) \hat{F}\left(z^{\prime}, \tau^{\prime}\right) \\
& -i n \kappa_{1} \int_{0}^{\tau} d \tau^{\prime} e^{-\Gamma\left(\tau-\tau^{\prime}\right)} \hat{E}_{q}\left(\tau^{\prime}\right) E_{s}\left(\tau^{\prime}\right) \sqrt{\frac{L}{a(\tau)-a\left(\tau^{\prime}\right)}} J_{1}\left(2 \sqrt{a(\tau)-a\left(\tau^{\prime}\right) L}\right)
\end{aligned}
$$

Eq.(3) is the main result of this Letter. The first term represents the decaying memory of the initial atomic coherence in the sample, the second term is the contribution from the Langevin noise associated with the decay of the coherence, and the last term represents the contribution from the absorbed quantum light. It is thus the last term, that describes the quantum memory capability of the atomic system. Note that the strong classical field $E_{s}\left(\tau^{\prime}\right)$ can be turned on and off, so that only the value of the quantum field in a certain time window is mapped onto the atomic system, where it is subsequently kept. We assume that the rate $\Gamma$ is dominated by the power broadening contribution $\Gamma_{s}$ when the classical field is turned on, and it can be quite small $\Gamma=\Gamma_{0}$ when the classical field is turned off to ensure long storage times. If the quantum field pulse $\hat{E}_{q}(\tau)$ and the overlapping classical pulse $E_{s}(\tau)$ are long enough so that $\Gamma \tau \gg 1$ the initial atomic state decays and the state determined by $\hat{E}_{q}(\tau)$ emerges instead. After the light pulses are turned off, the atomic "memory" state decays slowly with the rate $\Gamma_{0}$.

As an example of storing a quantum feature of light in atoms let us consider storing a squeezed state, which plays an important role in quantum information with continious variables 洵. For infinitely broadband squeezed light the quadrature operator $\hat{X}_{q}(z, \tau)=\operatorname{Re} \hat{E}_{q}(z, \tau)$ on the entry face of the sample can be written as $\left\langle\hat{X}_{q}(0, \tau) \hat{X}_{q}\left(0, \tau^{\prime}\right)\right\rangle=$ $2 \pi \hbar \omega / c\left\langle X_{0}^{2}\right\rangle \delta\left(\tau-\tau^{\prime}\right)$, where $\left\langle X_{0}^{2}\right\rangle$ is the dimensionless light noise, $\left\langle X_{0}^{2}\right\rangle=1$ in the case of broad band vacuum. In steady-state the variance of the atomic noise $\hat{X}=\operatorname{Re} \hat{\mathcal{Q}}_{L}$ becomes

$$
\begin{aligned}
\left\langle X^{2}\right\rangle & =n L\left(e^{-\alpha}\left(I_{0}(\alpha)+I_{1}(\alpha)\right)\right) \\
& +n L\left\langle X_{0}^{2}\right\rangle\left(1-e^{-\alpha}\left(I_{0}(\alpha)+I_{1}(\alpha)\right)\right)
\end{aligned}
$$

where $\alpha=a L / \Gamma$ is the optical depth of the sample, $a=\kappa_{1}^{*} \kappa_{2}\left|E_{s}\right|^{2}$ and $I_{0}$ and $I_{1}$ are Bessel functions of the first kind. In the case of vacuum incident on the sample we recover the atomic vacuum noise $\left\langle X^{2}\right\rangle=n L$, the number of atoms per unit area. The second term in (4), represents the light contribution to atomic noise, it is reduced when the light is squeezed, and in the case of ideally squeezed light $\left\langle X_{0}^{2}\right\rangle=0$ only the first term contributes to the atomic noise variance. We define the dimensionless expression in the parenthesis as a mapping efficiency for the Gaussian fields $\eta=\left(1-\left\langle X^{2}\right\rangle / n L\right) /\left(1-\left\langle X_{0}^{2}\right\rangle\right)$ (for ideally squeezed light $1-\eta$ quantifies the amount of spin squeezing). The results are plotted in Fig.1 (solid line) as a function of the optical depth $\alpha$. Storing squeezing in atoms with an efficiency higher than $90 \%$ requires an atomic sample with an optical depth of the order of $\gtrsim 60$. Note that by absorption of EPR beams in separate atomic samples, we may, e.g., prepare entangled atomic gases, see also [5]. If $\Gamma \approx \Gamma_{s}$, and the decoherence is dominated by the strong field that is required for the operational memory, then $\alpha \simeq(3 / 2 \pi) \lambda^{2} n L$, i.e., the optical depth is the same as for a resonant narrowband field. The dependence on the optical depth arises because the more squeezed light is absorbed in the sample, the more the atoms become squeezed. If only a fraction of the light field is absorbed, the atomic spins will not only be correlated with each other but also with the field leaving the sample, and thus the squeezing will be degraded, see also [6].

Various schemes for quantum state exchange between light and atoms based on cavity QED Raman-type interactions have been proposed in the past [7]9]. Quantum memory with a microwave cavity field as storage medium has been demonstrated in [10]. The fact, that the present proposal does not utilize high finesse cavities significantly simplifies the experimental realization. The above result can be compared with the proposal [11] and its experimental verification 
12. for squeezing the collective spin of an optically thick sample of $V$-type excited atoms via the interaction with squeezed light. As opposed to the theoretical bound of $50 \%$ mapping efficiency found in 11] the present proposal offers in principle a perfect transfer of the state of light onto atoms.

A steady state analysis in frequency domain similar to that in [11] leads to the following expression for the spectral collective atomic spin operator

$$
\begin{aligned}
\tilde{\mathcal{Q}}_{L}(\Delta) & =-\frac{i n}{\kappa_{2} E_{s}}\left(1-e^{i k(\Delta) L}\right) \tilde{E}_{q}(\Delta) \\
& +\int_{0}^{L} d z \frac{n}{\Gamma-i \Delta} e^{i k(\Delta)(L-z)} \tilde{F}(z, \Delta)
\end{aligned}
$$

where $\Delta$ is the detuning from the two-photon resonance and $k(\Delta)$ is the Lorentzian absorption profile $i k(\Delta)=$ $-a /(\Gamma-i \Delta)$. The atomic noise variance $\left\langle X^{2}\right\rangle=\int d \Delta \tilde{X}(\Delta) \tilde{X}(-\Delta)$ gives the same result as Eq.(任).

The simplest approach to quantum field propagation in a medium is the model of scattering by a collection of frequency-dependent beam splitters [13]. Each beam splitter removes a small fraction of a propagating light beam and it simultaneously couples in a small fraction of vacuum into the beam. The result for the noise spectrum of the transmitted light in our model coincides with such a simplified treatment and is given by

$$
\left\langle\tilde{X}^{2}(\Delta)\right\rangle=\left\langle\tilde{X}_{0}^{2}(\Delta)\right\rangle e^{-\frac{a \Gamma L}{\Gamma^{2}+\Delta^{2}}}+\left(1-e^{-\frac{a \Gamma L}{\Gamma^{2}+\Delta^{2}}}\right)
$$

For infinite bandwidth squeezed incident light this spectrum approaches the vacuum value 1 , for the frequencies where light is strongly attenuated. The width of this noise region grows with optical depth of the system. It is within this spectral region that quantum features of the light field are transferred onto atoms.

In the case of the finite bandwidth of ideal squeezing $114\left\langle\hat{X}_{q}(0, \tau) \hat{X}_{q}\left(0, \tau^{\prime}\right)\right\rangle \simeq 2 \pi \hbar \omega / c\left(\delta\left(\tau-\tau^{\prime}\right)-\Gamma_{q} / 2 e^{-\Gamma_{q}\left|\tau-\tau^{\prime}\right|}\right)$, calculations based on either Eq.(3) or Eq.(5) have to be carried out numerically and the mapping efficiencies for different spectral widths of squeezing $\Gamma_{q}$ are shown in Fig.1. We observe in the figure that when the entire bandwidth of squeezed light is completely absorbed in the sample, further growth of the optical depth leads only to the reduction of the spin squeezing, because the atoms which are not reached by the squeezed light are subject to the standard vacuum noise.

The macroscopic number of atoms in our atomic sample, of which most remain in the ground state, allows us to replace the sum of fermionic atomic operators by an effective bosonic operator $\hat{\mathcal{Q}}_{L}$ matching the bosonic operator of the light field. This restriction should be kept in mind when comparing our results to other analyses of spin-squeezing [15].

A suitable experimental setup for realization of the storage of field correlations in atoms is the cold atom fountain, e.g. as used in a frequency standard. A recent paper [16] reports operation of a laser cooled cesium fountain clock in the quantum limited regime meaning that the variance $\left\langle X^{2}\right\rangle=n L$ of the collective atomic spin associated with the $F=4, m=0-F=3, m=0$ two level system has been achieved. This means that the setup is suitable for the observation of squeezing of $\left\langle X^{2}\right\rangle$. The decoherence time $\Gamma_{0}$ of the order of a second reached in the atomic standard setup in principle allows quantum memory on this time scale. We thus propose to prepare atoms in the $F=3, m=0$ state (our state 1 , the level $F=4, m=0$ plays the role of our state 3 ) and to illuminate them by a Raman pulse containing the squeezed vacuum and the strong field as described above. After the pulse and after some delay the atoms are interrogated in a microwave cavity where their collective spin state is analyzed to verify that the memory works.

We now wish to address the experimental requirements for our proposal. For our two-level analysis to be valid, we

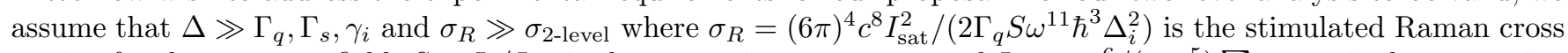
section for the quantum field, $S=I_{s} / I_{\text {sat }}$ is the saturation parameter and $I_{\text {sat }}=\omega^{6} /\left(9 \pi c^{5}\right) \sum \mu_{1 i} \mu_{3 i}$ is the saturation intensity for the strong field for $1 \leftrightarrow i, 3 \leftrightarrow i$ transitions, $\sigma_{\text {2-level }}=3 \lambda^{2} \gamma_{i}^{2} /\left(8 \pi \Delta_{i}^{2}\right)$ is the spontaneous 2-level cross section. In order to carry out the steady state solution of (1a) we assume $\Gamma_{s} \gg \tau_{\text {pulse }}^{-1}$ - where $\tau_{\text {pulse }}$ is the duration of the Raman pulse. Finally, the condition on the bandwidth of the quantum field $\Gamma_{q} \gg \tau_{\text {pulse }}^{-1}$ ensures that the pulse is long enough to contain all relevant correlations of the quantum state of the field. It is possible to satisfy all those conditions with the following set of parameters: $\Gamma_{q}=10^{7} \mathrm{~Hz}, \Delta=10^{9} \mathrm{~Hz}, S>4, \tau_{\text {pulse }}=10 \mathrm{msec}$. With the resonant optical depth of 20 achievable for $5 \times 10^{5}$ atoms a mapping efficiency exceeding $80 \%$ is possible (Fig.11). After the pulse is switched off the memory time $\Gamma_{0}^{-1}$ is set by the free evolution of the $F=4, m=0-F=3, m=0$ system and as mentioned above it can be as long as a second.

We have analyzed the possibility to transfer (write-down) a quantum state of light onto an atomic sample. And we have suggested how to perform a delayed measurement of the quantum state. We will now briefly discuss how to 
map the atomic state back onto a light field by interspecies teleportation [17]. To realize an effective teleportation of an atomic collective spin onto a light beam we suggest an approach similar to teleportation of light 18 20] with EPR correlated light beams and a beam-splitter type interaction between one of the beams and the atomic collective spin. Making a homodyne measurement of the light quadrature and a Ramsey measurement of the atomic spin we may employ the protocol used for light teleportation 18 20] and restore the atomic state in the other light beam.

To realize the "beam-splitter" we send a short pulse $\left(\tau_{\text {pulse }} \Gamma \ll 1\right.$ - so that dissipation processes do not take place) of one of the EPR beams through our atomic sample in the small optical depth regime $\left(\alpha \rightarrow a \tau_{\text {pulse }} L \ll 1\right)$. In our scheme the switching from high to small optical depth is made simply by adjusting the intensity of the coupling field $E_{s}$. In the weak coupling regime (small optical depth) the interaction between light and atoms (2b) - (3) can be described by a linear approximation leading to a "beam-splitter" type interaction. Introducing a new rescaled atomic operator $\hat{q}=(n L)^{-1 / 2} \hat{\mathcal{Q}}_{L}$ and the field "area" operator $\hat{\theta}=\sqrt{\lambda / 2 \pi \hbar \tau_{\text {pulse }}} \int_{0}^{\tau_{\text {pulse }}} d \tau^{\prime} \hat{E}_{q}\left(\tau^{\prime}\right)$ we obtain:

$$
\begin{aligned}
& \hat{q}_{\text {out }}=\hat{q}_{\text {in }}-i r \hat{\theta}_{\text {in }} \\
& \hat{\theta}_{\text {out }}=\hat{\theta}_{\text {in }}-i r \hat{q}_{\text {in }}
\end{aligned}
$$

The condition for such a linearization is a weak interaction, hence our "beam-splitter" is highly asymmetric, $r=$ $\sqrt{\alpha}=\sqrt{\sigma_{R} \tau_{\text {pulse }} L / \Gamma_{q}} \ll 1$. Teleportation with asymmetric beam splitters is possible but it requires a higher degree of correlation in the EPR beams. A simple estimate suggests, that the residual noise in the EPR pair must be smaller than $r$. If one assumes a stronger coupling in order to approach the symmetric beam-splitter case, the field probes a component of the atomic coherence, which deviates from the uniform integral in Eq.(3) due to the spatial variation of the probe light. If, for example, the probe is damped by a factor of order 2, it is reasonable to decompose the probed atomic coherence as a roughly even mixture of the uniform integral $\hat{\mathcal{Q}}_{L}$ and a "noise" operator which we, for simplicity may assume to be the standard vacuum noise. This noise is comparable to the "quduty" of noise [19] of a direct detection of the atomic ensemble and reconstruction of a corresponding field state ("classical teleportation").

We are grateful to Prof. Sam Braunstein for stimulating discussions of the atomic teleportation. This research has been funded by the Danish Research Council and by the Thomas B. Thriges Center for Quantum Information. AK acknowledge support of the ESF-QIT programme.

[1] G. Brassard, C. Crépeau, D. Mayers, and L. Salvail, quant-ph/9806031; D. Mayers, Phys. Rev. Lett. 78, 3414 (1997); H.-K. Lo and H. F. Chau, Phys. Rev. Lett. 78, 3410 (1997).

[2] M. G. Raymer and J. Mostowski, Phys. Rev. A 24, 1980 (1981).

[3] M. G. Raymer, I. A. Walmsley, J. Mostowski, and B. Sobolewska, Phys. Rev. A 32, 332 (1985).

[4] S. L. Braunstein, Nature 394, 47 (1998).

[5] E. S. Polzik, Phys. Rev. A 59, 4202 (1999).

[6] K. Mølmer, Eur. Phys. J. D 5, 301 (1999).

[7] J. I. Cirac, P. Zoller, H. J. Kimble, and H. Mabuchi, Phys. Rev. Lett. 78, 3221 (1997).

[8] A. S. Parkins and H. J. Kimble, J. Opt. B: Quantum Semiclass. Opt. 1, 496 (1999).

[9] H. Zeng and F. Lin, Phys. Rev. A 50, R3589 (1994); H. Zeng and F. L. W. Zhang, Phys. Lett. A 201,397 (1995).

[10] X. Maitre et al., Phys. Rev. Lett. 79, 769 (1997).

[11] A. Kuzmich, K. Mølmer, and E. S. Polzik, Phys. Rev. Lett. 79, 4782 (1997).

[12] J. Hald, J. L. Sørensen, C. Schori, and E. S. Polzik, Phys. Rev. Lett. 83, 1319 (1999).

[13] J. R. Jeffers, N. Imoto, and R. Loudon, Phys. Rev. A 47, 3346 (1993); R. Matloob, R. Loudon, S. M. Barnett, and J. Jeffers, Phys. Rev. A 52, 4823 (1995).

[14] J. H. Shapiro and K.-X. Sun, J. Opt. Soc. Am. B 11, 1130 (1994); C. W. Gardiner, Phys. Rev. Lett. 56, 1917 (1986).

[15] D. J. Wineland, J. J. Bollinger, W. M. Itano, and D. J. Heizen, Phys. Rev. A 50, 67 (1994); D. J. Wineland et al., Phys. Rev. A 46, R6797 (1992); M. Kitagawa and M. Ueda, Phys. Rev. Lett. 67, 1852 (1991).

[16] G. Santarelli et al., Phys. Rev. Lett. 82, 4619 (1999).

[17] C. S. Maierle, D. A. Lidar, and R. A. Harris, Phys. Rev. Lett. 81, 5928 (1998).

[18] A. Furusawa et al., Science 282, 706 (1998).

[19] S. L. Braunstein and H. J. Kimble, Phys. Rev. Lett. 80, 869 (1998).

[20] L. Vaidman, Phys. Rev. A 49, 1473 (1994). 


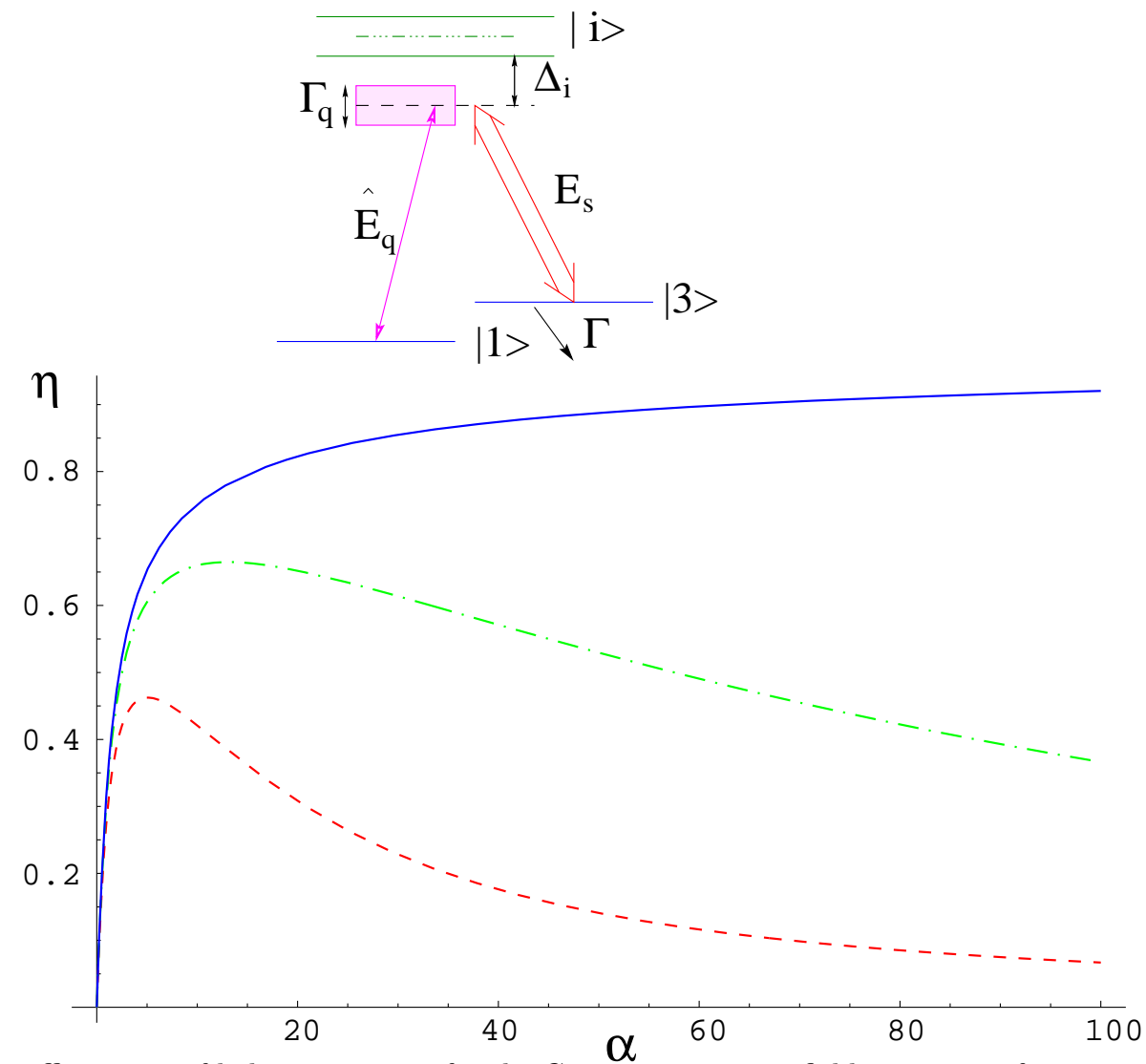

FIG. 1. Mapping efficiency $\eta$ of light onto atoms for the Gaussian quantum field states as a function of optical depth: solid line - infinite band squeezing, dash-dotted line $\Gamma_{q} / \Gamma=50$, dashed line $\Gamma_{q} / \Gamma=10$. Inset - Schematic representation of atomic levels. 